\title{
APOBEC3B deletion polymorphism and lung cancer risk in the southern Chinese population
}

\author{
Xiaosong Ben ${ }^{1 *}$, Dan Tian ${ }^{1 \#}$, Jiayu Liang ${ }^{2 *}$, Min Wu ${ }^{2 *}$, Fan $\mathrm{Xie}^{2}$, Jinlong Zheng ${ }^{2}$, Jingmin Chen ${ }^{2}$, \\ Qiaoyuan $\mathrm{Fei}^{2}$, Xinrong Guo ${ }^{2}$, Xueqiong Weng ${ }^{2}$, Shan $\mathrm{Liu}^{2}$, Xin $\mathrm{Xie}^{2}$, Yuting Ying ${ }^{2}$, Guibin Qiao ${ }^{1}$, \\ Chunxia Jing ${ }^{2,3}$
}

${ }^{1}$ Department of Thoracic Surgery, Guangdong Provincial People's Hospital, Guangdong Academy of Medical Sciences, Guangzhou, China; ${ }^{2}$ Department of Epidemiology, School of Medicine, Jinan University, Guangzhou, China; ${ }^{3}$ Guangdong Key Laboratory of Environmental Pollution and Health, Jinan University, Guangzhou, China

Contributions: (I) Conception and design: C Jing, G Qiao; (II) Administrative support: X Ben, D Tian, J Liang, M Wu; (III) Provision of study materials or patients: X Ben, D Tian; (IV) Collection and assembly of data: J Liang, M Wu, F Xie, J Zheng, J Chen, Q Fei; (V) Data analysis and interpretation: J Liang, M Wu, X Weng, S Liu, X Xie, Y Ying; (VI) Manuscript writing: All authors; (VII) Final approval of manuscript: All authors.

"These authors contributed equally to this work.

Correspondence to: Guibin Qiao. Department of Thoracic Surgery, Guangdong Provincial People’s Hospital, Guangdong Academy of Medical Sciences, Guangzhou 510080, China. Email: guibinqiao@126.com; Chunxia Jing. Department of Epidemiology, School of Medicine, Jinan University, No. 601 Huangpu Ave West, Guangzhou 510632, China. Email: jcxphd@gmail.com.

Background: Approximately $80-85 \%$ of lung cancer is the non-small cell lung cancer (NSCLC) subtype, which ranks as the leading cause of cancer deaths worldwide. APOBEC3B (A3B) was reported to be a key source of mutations in NSCLC. However, the role of the A3B deletion polymorphism in the etiology of NSCLC has not been well-documented.

Methods: A case-control study with 317 NSCLC patients and 334 healthy controls was conducted to explore the association between the A3B deletion polymorphism and the risk of NSCLC. The unconditional logistic regression model was performed to calculate the odds ratio (OR) and the $95 \%$ confidence interval (CI), and the confounding factors were adjusted, including age, gender, and smoking status, to estimate the risk. An analysis of gene-environment interactions was performed using multifactor dimensionality reduction (MDR) software.

Results: We found that the del/del genotype of A3B deletion significantly increased NSCLC risk. Compared with individuals carrying the ins/ins genotype of $\mathrm{A} 3 \mathrm{~B}$ deletion, individuals with the del/del genotype had a 2.36 times increased risk of developing NSCLC after adjusting for confounding factors (OR =2.71, 95\% CI: 1.67-4.42, $\mathrm{P}<0.001$ ). A 3-factor gene-environment (A3B deletion, gender, and smoking) interaction model was found for NSCLC (OR =4.407, 95\% CI: 1.174-16.549, P=0.028).

Conclusions: We propose that the A3B deletion polymorphism can increase the risk of developing NSCLC, and their interactions with gender and smoking may contribute to the risk of NSCLC in the southern Chinese population.

Keywords: Non-small cell lung cancer (NSCLC); APOBEC3B deletion (A3B deletion); polymorphism; interaction; association

Submitted Nov 05, 2020. Accepted for publication Apr 17, 2021.

doi: 10.21037/atm-21-989

View this article at: http://dx.doi.org/10.21037/atm-21-989 


\section{Introduction}

Lung cancer ranks as the most commonly diagnosed cancer. and the leading cause of cancer death worldwide, with an estimated 2,093,876 new cases and 1,761,007 deaths in 2018 (1). Small cell lung cancer (SCLC) and non-small cell lung cancer (NSCLC) are the 2 main histological subtypes of lung cancer, and approximately $80-85 \%$ of lung cancers are NSCLC, including adenocarcinoma, squamous cell carcinoma, and large-cell carcinoma (2). Although medical technology has developed rapidly and has significantly improved the survival of lung cancer patients, the etiology of lung cancer remains unclear and prognosis is still poor for some patients, necessitating further investigations. Smoking, as one of the most well-known environmental risk factors, is a major contributor to lung cancer development. According to the Global Burden of Disease, in 2017, approximately $63 \%$ of lung cancer deaths in China were attributable to smoking (3). Furthermore, there is also a genetic component to lung cancer (4), and heritability estimates of the genetic risk for lung cancer are approximately $8 \%$ (5). Smoking can also cause gene mutations which may lead to a heavy mutation load of lung cancer $(6,7)$. Therefore, while smoking is a clear risk factor, the genetic factors also cannot be ignored.

The apolipoprotein B mRNA-editing catalytic polypeptide-like 3 (A3) family (8), which plays a crucial role in antiviral innate cellular immunity, consists of 7 members [A3A, APOBEC3B (A3B), A3C, A3DE, A3F, A3G, and $\mathrm{A} 3 \mathrm{H}$ ] on chromosome 22 (9). They can cause deaminations of cytosine to uracil in DNA via an enzyme called activationinduced cytidine deaminase (10), leading to DNA damage. Studies have shown that the activity of A3A and A3B are linked to cancer. Recent research has also shown that the expression levels of $\mathrm{A} 3 \mathrm{~B}$ have a strong positive correlation with tumor mutation loads (11), along with a strong A3B upregulation in the majority of tumor types, including breast, uterus, bladder, head and neck, and lung (adeno- and squamous cell carcinomas) (12), suggesting that A3B may be a general endogenous mutagen that contributes to human cancers. Patients with a higher level of A3B gene expression in their tumors had a lower survival rate as compared to patients with lower expression A3B gene expression in their tumors (13-15).

Recently, a $29.5 \mathrm{~kb}$ germline deletion in the A3 gene region has been found to be associated with the risk of breast cancer (11), colon cancer (16), cervical cancer (17), lung cancer (16), prostate cancer (16), ovarian cancer (18), bladder cancer (19), oral cancer (17), and hepatocellular carcinoma (20). This $29.5 \mathrm{~kb}$ deletion removes the 3 ' end of the $\mathrm{A} 3 \mathrm{~A}$ gene and a large part of the $\mathrm{A} 3 \mathrm{~B}$ gene, creating a hybrid gene that transcribes mRNA with the A3A coding region and the A3B 3'-untranslated regions (UTR) (19), and the amino acid sequence of this fusion protein is identical to APOBEC3A (21). Interestingly, carriers of the A3B deletion transcript were shown to have higher A3A mRNA stability, resulting in higher intracellular levels and more severe DNA damage (22). Among all the members of the A3 family, A3A can hypermutate DNA by inducing DNA double-strand breaks $(23,24)$, suggesting $\mathrm{A} 3 \mathrm{~A}$ has a role in pro-inflammatory conditions that might cause cancer (25). The $A 3 B$ germline deletion allele also causes deletion of the $\mathrm{A} 3 \mathrm{~B}$ coding sequence, and thus, the absence of $\mathrm{A} 3 \mathrm{~B}$ in homozygous patients (21). Evidence also has supported the association between A3B upregulation and NSCLCrelated processes (26). However, the mechanism of A3B overexpression and the $\mathrm{A} 3 \mathrm{~B}$ deletion polymorphism in human tumorigenesis remains to be determined. Moreover, ethnic differences have been observed between the A3B deletion polymorphism and their association with the occurrence of cancer. The frequency of the A3B deletion variant was shown to be $6 \%$ among individuals of European descent, 37\% among individuals of Asian descent, and $57.7 \%$ among individuals of American descent (27).

The relationship between $\mathrm{A} 3 \mathrm{~B}$ gene deletion and the risk of non-small cell lung cancer in the Chinese population has not been studied yet, and the effects of interaction between A3B deletion polymorphism and environmental factors on the risk of NSCLC remain unclear. To identify the A3B deletion polymorphism relevant to NSCLC, we investigated the genotype distribution of A3B deletion in NSCLC and explored their association with NSCLC risk to provide novel biomarkers for early prevention and prognosis of NSCLC in the southern Chinese population. We present the following article in accordance with the STROBE reporting checklist (available at http://dx.doi.org/10.21037/ atm-21-989).

\section{Methods}

\section{Ethical statement}

All subjects were supplied with written informed consent forms for both clinical epidemiological investigations and blood sample collection before enrolment. The patient or family involved in the study provided informed 


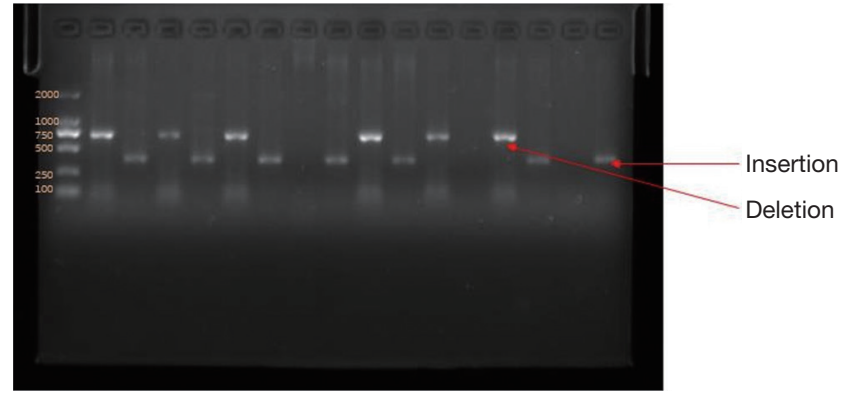

Figure 1 The results of the agarose gel electrophoresis.

consent. This study was conducted in accordance with the recommendations of the Helsinki Declaration (as revised in 2013), and the ethics committee of Guangdong Provincial People's Hospital approved the study protocol.

\section{Study subjects and data collection}

This case-control study was conducted based on the population from Guangzhou, Guangdong Province. A total of 317 NSCLC cases and 334 controls were included in this study. The inclusion criteria for the cases were as follows: (I) diagnosed as NSCLC by histopathological examination; (II) they had not undergone previous systemic therapy for metastatic disease; (III) had an Eastern Cooperative Oncology Group (ECOG) performance status score of 0 or 1; (IV) had at least 1 measurable lesion according to Response Evaluation Criteria in Solid Tumors (RECIST), version $1.1(28,29)$. The control group consisted of healthy people who came to the hospital for medical examinations, or patients without cancer or other diseases that might have been related to lung cancer. In order to obtain their demographic characteristics and information on environmental exposures, we conducted a questionnaire survey. EDTA anticoagulation tubes $(3-5 \mathrm{~mL})$ were used to collect peripheral blood, and the blood was stored at $-80^{\circ} \mathrm{C}$. Participants who smoked less than 100 cigarettes in their lifetime were defined as non-smokers, otherwise, they were classified as smokers.

Power analysis was performed for this study by Power and Sample Size (PASS 15). The sample size for $90.1 \%$ power value is 330 persons in total, and our sample size has been a total of 651 subjects.

\section{Genotype analysis of the A3B deletion polymorphism}

The Blood DNA Kit (OMEGA) was used to extract peripheral blood DNA according to the manufacturer's protocol, and the samples were stored at $-20^{\circ} \mathrm{C}$. We then used a TaKaRa Premix Taq kit to perform the PCR. Insertion primers amplified the insertion sequence configuration and generation of the $490 \mathrm{bp}$ PCR product. Deletion primers amplified the deletion sequence configuration and generation of the $700 \mathrm{bp}$ PCR product. The genotype primers included insertion forward primer: TTGGTGCTGCCCCCTC, reverse primer, TAGAGACTGAGGCCCAT; deletion forward primer, TAGGTGCCACCCCGAT, reverse primer, TTGAGCATAATCTTACTCTTGTAC. The $25 \mu \mathrm{L}$ reaction volume included $2 \mu \mathrm{L}$ primer $\mathrm{F}$ and $2 \mu \mathrm{L}$ primer $\mathrm{R}$, $2 \mu \mathrm{g}$ DNA, $12.5 \mu \mathrm{L}$ Premix Taq, and $6.5 \mu \mathrm{L}$ sterilized water. The conditions of PCR were as follows: $95.0^{\circ} \mathrm{C}$ for $5 \mathrm{~min}$, followed by 40 cycles at $95.0^{\circ} \mathrm{C}$ for $5 \mathrm{~min}, 61.0^{\circ} \mathrm{C}$ for $1 \mathrm{~min}$, and $72.0{ }^{\circ} \mathrm{C}$ for $1 \mathrm{~min}$, extension for $7 \mathrm{~min}$ at $72.0{ }^{\circ} \mathrm{C}$, with a final step at $4.0^{\circ} \mathrm{C}$. Agarose gel electrophoresis was employed to analyze the PCR products (Figure 1).

Among the 334 controls, 152 were homozygous for the A3B insertion allele, accounting for $45.51 \%$, while $138(41.32 \%)$ were heterozygous, and $44(13.17 \%)$ were homozygous for the deletion allele. This resulted in an observed minor allele frequency (MAF) of 1.989 , and the genotype distribution did not deviate from the HardyWeinberg equilibrium $(\mathrm{P}>0.05)$.

\section{Statistical analysis}

The demographic data and genotype characteristics are presented as number (percentage), and the mean \pm SD. For categorical variables, the $\chi^{2}$-test was performed to calculate the differences between demographic variables and genotype distribution of A3B deletion between the case group and the control group. For continuous variables with a normal distribution, an ANOVA was performed (Table 1 and Table S1). Logistic regression analyses were used to calculate the odds ratios (ORs), the $95 \%$ confidence intervals (CIs), and to estimate the associations between A3B deletion and NSCLC risk. Model 1 was unadjusted. Model 2 was adjusted for age, gender, and smoking status (Table 2). The Hardy-Weinberg equilibrium (HWE) of the control group was calculated by $\chi^{2}$ statistics. Statistical significance was set as $\mathrm{P}<0.05$, and all tests were two-sided. All of the statistical analyses were performed using STATA.

The multifactor dimensionality reduction (MDR) software was used to identify the best gene-environment interaction model of A3B deletion. The environmental 
Table 1 The baseline population characteristics of the control group and NSCLC patients

\begin{tabular}{lccc}
\hline Characteristic & Case & Control & $\chi^{2}$ \\
\hline Age & & $226(67.46)$ & 72.694 \\
$<55$ & $108(34.07)$ & $109(32.54)$ & 26.763 \\
$\geq 55$ & $209(65.93)$ & & $<0.001$ \\
Gender & & $117(35.03)$ & \\
Male & $175(55.21)$ & $217(64.97)$ & 7.923 \\
Female & $142(44.79)$ & & 0.001 \\
Smoking & & $260(83.60)$ & \\
Yes & $236(74.45)$ & $51(16.40)$ & \\
No & $81(25.55)$ & $152(45.51)$ & $<0.001$ \\
Genotype & $126(39.75)$ & $138(41.32)$ & \\
Wildtype (II) & $105(33.12)$ & $44(13.17)$ & \\
Hetero (ID) & $86(27.13)$ & & \\
Deletion (DD) & & & \\
\hline
\end{tabular}

NSCLC, non-small cell lung cancer.

Table 2 Association between the A3B deletion polymorphism and NSCLC

\begin{tabular}{|c|c|c|c|c|c|c|c|}
\hline Genotype & \multicolumn{2}{|c|}{ Case } & \multicolumn{2}{|c|}{ Control } & OR & $\mathrm{P}$ value & $95 \% \mathrm{Cl}$ \\
\hline \multicolumn{8}{|l|}{ Model 1} \\
\hline Wildtype (II) & 126 & 39.75 & 152 & 45.51 & Reference & & \\
\hline Hetero (ID) & 105 & 33.12 & 138 & 41.32 & 0.92 & 0.628 & $0.65-1.30$ \\
\hline \multicolumn{8}{|l|}{ Model 2} \\
\hline Wildtype (II) & 126 & 39.75 & 141 & 45.48 & Reference & & \\
\hline Hetero (ID) & 105 & 33.12 & 132 & 42.58 & 0.87 & 0.466 & $0.59-1.27$ \\
\hline Deletion (DD) & 86 & 27.13 & 37 & 11.94 & 2.71 & $<0.001$ & $1.67-4.42$ \\
\hline
\end{tabular}

Model 1: unadjusted model; Model 2: adjusted for gender, age, smoking. NSCLC, non-small cell lung cancer; OR, odds ratio; Cl, confidence interval.

factors we included in the MDR were age, gender, and smoking status (Table 3).

\section{Results}

\section{Population characteristics}

The baseline characteristics of all included individuals are presented in Table 1. There were significant differences between the NSCLC group and the control group in age, gender, smoking, and genotype distribution $(\mathrm{P}<0.05)$. In the case group, the population over 55 years old accounted for $65.93 \%$, compared to only $32.54 \%$ in the control group. The case group was also $55 \%$ male, compared to $35.03 \%$ male in the control group. The probability of detecting the del/del genotype was higher in the case group than the control group $(27.13 \%$ vs. $13.17 \%, \mathrm{P}<0.001)$. 
Table 3 The gene-environment interaction model for NSCLC using MDR analysis

\begin{tabular}{lccc}
\hline Model & TBA & CVC & P value \\
\hline Genotype & 0.6004 & $10 / 10$ & 0.001 \\
Genotype*smoking & 0.6318 & $10 / 10$ & 0.001 \\
Genotype*gender*smoking & 0.6415 & $10 / 10$ & 0.001 \\
\hline
\end{tabular}

*, gene-environment interaction model. NSCLC, non-small cell lung cancer; MDR, multifactor dimensionality reduction.

\section{Association between the A3B deletion genotype and NSCLC}

A significant association between the A3B del/del genotype and the risk of NSCLC was identified after adjusting for confounding factors. The individuals carrying the del/del genotype were at a 2.71 times higher risk of developing NSCLC than those with the ins/ins genotype.

\section{Association between genotype distributions in NSCLC patients and clinical information}

The clinical information of the case group is shown in Table S1. However, we did not identify differences between clinical information (tumor size, lymph node metastasis, clinical stage) and genotype distribution.

\section{The interaction between A3B deletion genotypes and environmental factors}

To explore the roles of gene-environment interactions on the risk of NSCLC, generalized multifactor dimensionality reduction (GMDR) was conducted to analyze the interaction between A3B deletion genotypes and environmental factors in NSCLC. The results showed that the best interaction model of NSCLC was the 3-factor model, including A3B deletion genotype, gender, and smoking (Table 3).

Compared with the reference group (ins/ins genotype, female, and non-smoking), individuals who carried the A3B ins/del genotype and were females and non-smokers had a lower risk of NSCLC. Regardless of smoking status, males had a higher risk of developing NSCLC with the ins/del genotype (1.907 and 3.375), and the risk among smokers was higher. Among individuals who carried the del/del genotype, males had approximately 7.312- and 7.769-time increased risk of developing NSCLC, and smokers had a higher risk of NSCLC (Table 4, Figure 2).

\section{Discussion}

We conducted a hospital-based case-control study to determine whether the $\mathrm{A} 3 \mathrm{~B}$ deletion polymorphism increased NSCLC risk among the southern Chinese population. In this study, we observed that individuals carrying the del/del genotype in the A3B deletion polymorphism had a 2.71-fold higher risk of developing NSCLC than those with the ins/ins genotype. Our results are consistent with previous findings in Norway (16), suggesting that $\mathrm{A} 3 \mathrm{~B}$ deletion may be useful as a biomarker for screening NSCLC in the early stages. Using the best 3 -factor interaction model, we found that the A3B deletion polymorphism interacts with smoking and gender to increase the risk of developing NSCLC. To the best of our knowledge, this is the first study to explore the correlations between the A3B deletion polymorphism with an increased risk of NSCLC among the southern Chinese population. It therefore provides an important molecular epidemiological perspective on the correlations between the $\mathrm{A} 3 \mathrm{~B}$ deletion polymorphism and NSCLC.

Recent studies have reported that the A3B deletion polymorphism is associated with an increased risk of various cancer types, including breast (11,30,31), colon (16), cervical (17), lung (16), prostate (16), ovarian (18), bladder (19), oral (17), and hepatocellular carcinoma (20). A3 $\mathrm{B}$ deletion is also correlated with an increased mutational load (21). Apart from deleting the A3B coding frame, A3B deletion carriers also have the ability to generate a novel fusion transcript that is fused to the A3B 3'UTR (27). We are curious how this will affect the expression of $\mathrm{A} 3 \mathrm{~B}$. However, the expression of $\mathrm{A} 3 \mathrm{~B}$ is not only regulated by the $\mathrm{A} 3 \mathrm{~B}$ gene, the expression of $\mathrm{A} 3 \mathrm{~B}$ is also regulated by the p53 gene. P53 mutations up-regulated the expression of A3B (15). Consequently, A3A mRNA in the fusion transcript has been shown to become more stable (10- to 20-fold higher steady-state 
Table 4 Risk factor analysis of NSCLC based on 3 factors: genotype, gender, and smoking

\begin{tabular}{|c|c|c|c|c|c|c|}
\hline АРОВЕСЗВ & Gender & Smoking status & Case & Control & OR $(95 \% \mathrm{Cl})$ & $P$ value \\
\hline ins/ins & Female & + & 2 & 6 & $0.456(0.089-2.340)$ & 0.347 \\
\hline ins/ins & Male & - & 32 & 23 & $1.762(0.975-3.188)$ & 0.061 \\
\hline ins/ins & Male & + & 26 & 22 & $1.620(0.841-3.120)$ & 0.149 \\
\hline ins/del & Female & + & 3 & 4 & $1.028(0.222-4.760)$ & 0.972 \\
\hline ins/del & Male & - & 32 & 23 & 1.907 (1.018-3.574) & $0.044^{*}$ \\
\hline ins/del & Male & + & 32 & 14 & 3.375 (1.638-6.954) & $0.001^{*}$ \\
\hline $\mathrm{del} / \mathrm{del}$ & Female & - & 36 & 26 & $1.898(1.040-3.463)$ & $0.037^{*}$ \\
\hline $\mathrm{del} / \mathrm{del}$ & Male & + & 17 & 3 & 7.769 (2.034-28.510) & $0.002^{*}$ \\
\hline
\end{tabular}

${ }^{*}, \mathrm{P}<0.05$. NSCLC, non-small cell lung cancer; OR, odds ratio.

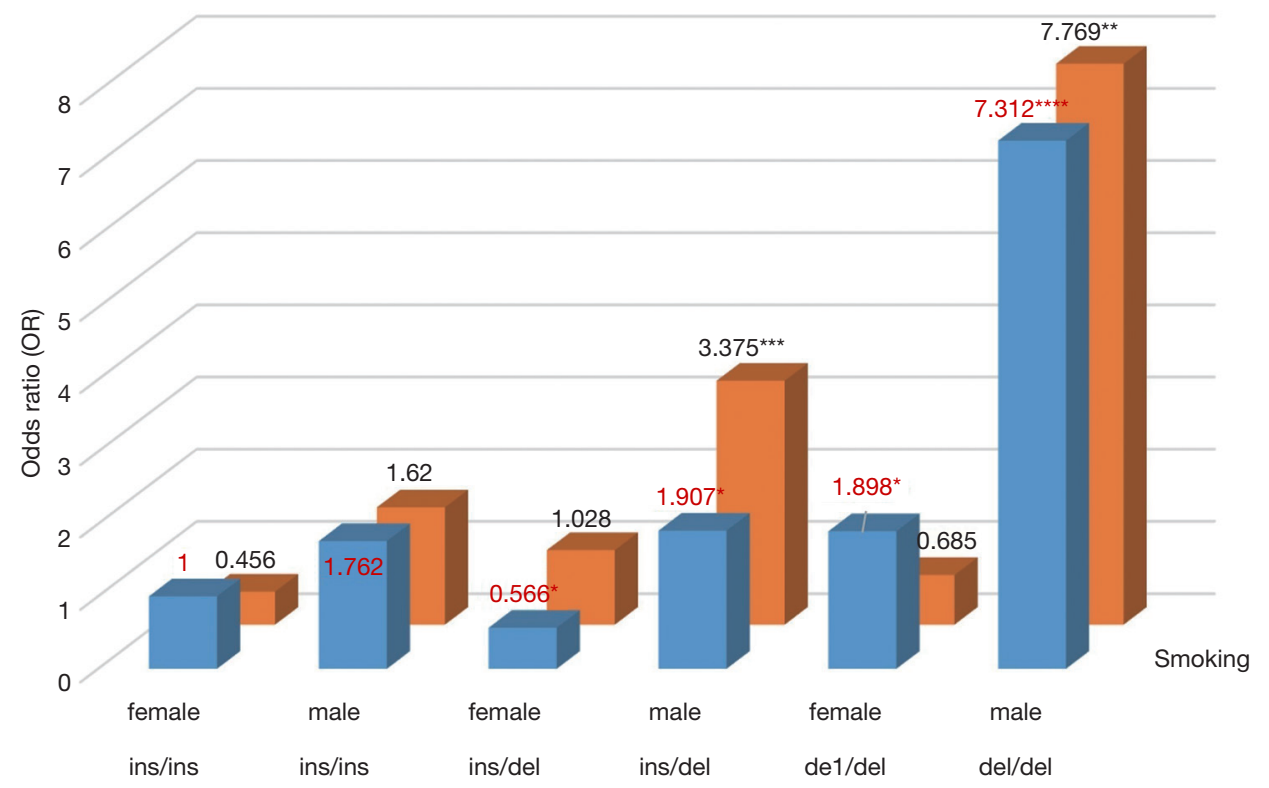

Figure 2 Risk factor analysis of gene-environment interactions in non-small cell lung cancer (NSCLC). Model: APOBEC3B deletion genotypes, gender, and smoking. The reference group was the interaction of ins/ins genotype for APOBEC3B, gender, and smoking status. The odds ratio (OR) value is shown in the figure. ${ }^{*} \mathrm{P}<0.05$ and ${ }^{* *} \mathrm{P}<0.01,{ }^{* * *} \mathrm{P}<0.001,{ }^{* * * *} \mathrm{P}<0.000$.

levels of $\mathrm{A} 3 \mathrm{~A}$ ), resulting in higher levels of $\mathrm{A} 3 \mathrm{~A}$ and more severe DNA damage (22). Since A3A is capable of acting as a hypermutator to break DNA, and is sensitive to inflammatory environments involving type I and II interferons, this can result in chronic inflammation which has long been associated with the onset of cancer (32). Moreover, repeated A3A-induced DNA damage is a major driving force behind iterative somatic mutations of the human genome (22). The frequency of the A3B deletion polymorphism varies across different populations, as it 
is rare in Africans and Europeans (0.9\% and 6\%), but more common in East Asians and Amerindians (36.9\% and $57.7 \%$ ) (27). In our southern Chinese population, $57.3 \%$ of individuals carried the A3B deletion allele. It may explain the heterogeneity in the associations between A3B deletion and cancer risk in different ethnicities. The associations stay consistent in Asian populations (17,33,34), while the associations are inconsistent in Europeans $(16,18,35)$.

Furthermore, we evaluated the association between the A3B deletion polymorphism and the clinical features of the 317 NSCLC patients. However, we found that the A3B deletion polymorphism was not significantly associated with lymph node metastasis number, tumor size, and clinical stage, which is consistent with a previous study in breast cancer (36). Gansmo et al. observed that lung cancer was associated with the deletion allele in Caucasian individuals under 50 years old, and the OR gradually decreased with increasing age (16). However, this observation was not reproduced in our research. We classify the age group with 55 years old as the boundary value, there is also no association between APOBEC $3 \mathrm{~A} / \mathrm{B}$ deletion and the age at diagnosis

Recently, it has been reported that susceptibility to NSCLC could be affected by various factors, including interactions between various environmental factors $(37,38)$ and different polymorphisms (39). Using the best 3 -factor interaction model, we observed that the $\mathrm{A} 3 \mathrm{~B}$ deletion polymorphism interacts with smoking and gender to increase the possibility of developing NSCLC. Since the A3B deletion polymorphism is a genetic factor, along with gender it cannot be altered. Hence, smoking is the only modifiable variable. As a result, by controlling smoking, how much can the risk of NSCLC based on these 3 characteristics be reduced? Smoking among males with the A3B del/del genotype was associated with a $5.9 \%$ increased risk of NSCLC $\left(\mathrm{OR}_{\mathrm{del} / \mathrm{del}+\text { smoking male }} 7.77 \mathrm{vs}\right.$. $\mathrm{OR}_{\mathrm{del} / \mathrm{del}+\text { non- }}$ smoking male: 7.31) compared to non-smoking females with the ins/ins genotype. Furthermore, males with the A3B ins/ del genotype had significantly increased NSCLC risk by $43.5 \%\left(\mathrm{OR}_{\mathrm{del} / \mathrm{del}+\text { smoking male }}: 3.38 v \mathrm{~s} . \mathrm{OR}_{\mathrm{del} / \mathrm{del}+\text { non-smoking male }}\right.$ : 1.91), compared to non-smoking females with the ins/ins genotype. This outcome is consistent with the analysis of the clinical data between healthy control individuals and NSCLC patients, which showed that smoking is related to lung tumorigenesis (40). However, due to the limited female smoker samples, the power to precisely predict the cancer risk of smoking females with the A3B deletion genotype is also limited.

There are some limitations in our study. First, the sample in the study was not large, which might have restricted the ability to explore weaker associations among NSCLC, environmental factors, and the A3B deletion polymorphism. What's more, our sample was selected from the southern population in China and cannot represent the whole population. Second, the clinical characteristics of the included individuals collected were not very comprehensive. Several prognostic characteristics were not collected, such as survival time and tumor recurrence status. In addition, we should have also included more meaningful environmental factors in our analysis, such as diet and passive smoke exposure. Therefore, further large and welldesigned investigations are needed in the future to support our findings.

\section{Acknowledgments}

Funding: This work was supported in part by Training Program of the Major Research Plan of the National Natural Science Foundation of China (Grant numbers: 91543132), National Natural Science Foundation of China (Grant numbers: 81541070, 81101267, and 30901249), Guangdong Natural Science Foundation (Grant numbers: 2018A030313601, 10151063201000036, S2011010002526 and 2016A030313089), Guangdong Province Medical Research Foundation (Grant number: A2014374 and A2015310) and Project from Jinan University (Grant number: 21612426, 21615426, JNUPHPM2016001, and JNUPHPM2016002).

\section{Footnote}

Reporting Checklist: The authors have completed the STROBE reporting checklist. Available at http://dx.doi. org/10.21037/atm-21-989

Data Sharing Statement: Available at http://dx.doi. org/10.21037/atm-21-989

Conflicts of Interest: All authors have completed the ICMJE uniform disclosure form (available at http://dx.doi. org/10.21037/atm-21-989). The authors have no conflicts of interest to declare.

Ethical Statement: The authors are accountable for all aspects of the work in ensuring that questions related 
to the accuracy or integrity of any part of the work are appropriately investigated and resolved. The patient or family involved in the study provided informed consent. This study was conducted in accordance with the recommendations of the Helsinki Declaration (as revised in 2013), and the ethics committee of Guangdong Provincial People's Hospital approved the study protocol.

Open Access Statement: This is an Open Access article distributed in accordance with the Creative Commons Attribution-NonCommercial-NoDerivs 4.0 International License (CC BY-NC-ND 4.0), which permits the noncommercial replication and distribution of the article with the strict proviso that no changes or edits are made and the original work is properly cited (including links to both the formal publication through the relevant DOI and the license). See: https://creativecommons.org/licenses/by-nc-nd/4.0/.

\section{References}

1. Bray F, Ferlay J, Soerjomataram I, et al. Global cancer statistics 2018: GLOBOCAN estimates of incidence and mortality worldwide for 36 cancers in 185 countries. CA Cancer J Clin 2018;68:394-424.

2. Travis WD. Pathology of lung cancer. Clin Chest Med 2002;23:65-81, viii.

3. Institute for Health Metrics and Evaluation. Global burden of disease 2017. 2017. Available online: http:// vizhub.healthdata.org/gbd-compare/\#. 2017.

4. Manolio TA, Collins FS, Cox NJ, et al. Finding the missing heritability of complex diseases. Nature 2009;461:747-53.

5. Liu P, Vikis HG, Wang D, et al. Familial aggregation of common sequence variants on 15 q24-25.1 in lung cancer. J Natl Cancer Inst 2008;100:1326-30.

6. Alexandrov LB, Ju YS, Haase K, et al. Mutational signatures associated with tobacco smoking in human cancer. Science 2016;354:618-22.

7. Lichtenstein P, Holm NV, Verkasalo PK, et al. Environmental and heritable factors in the causation of cancer--analyses of cohorts of twins from Sweden, Denmark, and Finland. N Engl J Med 2000;343:78-85.

8. Conticello SG. The AID/APOBEC family of nucleic acid mutators. Genome Biol 2008;9:229.

9. Jarmuz A, Chester A, Bayliss J, et al. An anthropoidspecific locus of orphan $\mathrm{C}$ to $\mathrm{U}$ RNA-editing enzymes on chromosome 22. Genomics 2002;79:285-96.

10. Refsland EW, Harris RS. The APOBEC3 family of retroelement restriction factors. Curr Top Microbiol Immunol 2013;371:1-27.

11. Cescon DW, Haibe-Kains B, Mak TW. APOBEC3B expression in breast cancer reflects cellular proliferation, while a deletion polymorphism is associated with immune activation. Proc Natl Acad Sci U S A 2015;112:2841-6.

12. Burns MB, Temiz NA, Harris RS. Evidence for APOBEC3B mutagenesis in multiple human cancers. Nature Genetics 2013;45:977-83.

13. Gara SK, Tyagi MV, Patel DT, et al. GATA3 and APOBEC3B are prognostic markers in adrenocortical carcinoma and APOBEC3B is directly transcriptionally regulated by GATA3. Oncotarget 2020;11:3354-70.

14. Roper N, Gao S, Maity TK, et al. APOBEC Mutagenesis and Copy-Number Alterations Are Drivers of Proteogenomic Tumor Evolution and Heterogeneity in Metastatic Thoracic Tumors. Cell Rep 2019;26:2651-66.e6.

15. Periyasamy M, Singh AK, Gemma C, et al. p53 controls expression of the DNA deaminase APOBEC3B to limit its potential mutagenic activity in cancer cells. Nucleic Acids Res 2017;45:11056-69.

16. Gansmo LB, Romundstad P, Hveem K, et al. APOBEC3A/ $\mathrm{B}$ deletion polymorphism and cancer risk. Carcinogenesis 2018;39:118-24.

17. Revathidevi S, Manikandan M, Rao AK, et al. Analysis of APOBEC3A/3B germline deletion polymorphism in breast, cervical and oral cancers from South India and its impact on miRNA regulation. Tumour Biol 2016;37:11983-90.

18. Klonowska K, Kluzniak W, Rusak B, et al. The $30 \mathrm{~kb}$ deletion in the APOBEC3 cluster decreases APOBEC3A and APOBEC3B expression and creates a transcriptionally active hybrid gene but does not associate with breast cancer in the European population. Oncotarget 2017;8:76357-74.

19. Middlebrooks CD, Banday AR, Matsuda K, et al. Association of germline variants in the APOBEC3 region with cancer risk and enrichment with APOBEC-signature mutations in tumors. Nat Genet 2016;48:1330-8.

20. Zhang T, Cai J, Chang J, et al. Evidence of associations of APOBEC3B gene deletion with susceptibility to persistent $\mathrm{HBV}$ infection and hepatocellular carcinoma. Hum Mol Genet 2013;22:1262-9.

21. Nik-Zainal S, Wedge DC, Alexandrov LB, et al. Association of a germline copy number polymorphism of APOBEC3A and APOBEC3B with burden of putative APOBEC-dependent mutations in breast cancer. Nat Genet 2014;46:487-91.

22. Caval V, Suspène R, Shapira $M$, et al. A prevalent 
cancer susceptibility APOBEC3A hybrid allele bearing APOBEC3B 3'UTR enhances chromosomal DNA damage. Nat Commun 2014;5:5129.

23. Suspene R, Aynaud MM, Guetard D, et al. Somatic hypermutation of human mitochondrial and nuclear DNA by APOBEC3 cytidine deaminases, a pathway for DNA catabolism. Proc Natl Acad Sci U S A 2011;108:4858-63.

24. Carpenter MA, Li M, Rathore A, et al. Methylcytosine and normal cytosine deamination by the foreign DNA restriction enzyme APOBEC3A. J Biol Chem 2012;287:34801-8.

25. Mussil B, Suspene R, Aynaud MM, et al. Human APOBEC3A isoforms translocate to the nucleus and induce DNA double strand breaks leading to cell stress and death. PLoS One 2013;8:e73641.

26. Wang S, Jia M, He Z, et al. APOBEC3B and APOBEC mutational signature as potential predictive markers for immunotherapy response in non-small cell lung cancer. Oncogene 2018;37:3924-36.

27. Kidd JM, Newman TL, Tuzun E, et al. Population stratification of a common APOBEC gene deletion polymorphism. PLoS Genet 2007;3:e63.

28. Eisenhauer EA, Therasse P, Bogaerts J, et al. New response evaluation criteria in solid tumours: revised RECIST guideline (version 1.1). Eur J Cancer 2009;45:228-47.

29. Lv X, Cui Z, Li H, et al. Polymorphism in lncRNA AC008392.1 and its interaction with smoking on the risk of lung cancer in a Chinese population. Cancer Manag Res 2018;10:1377-87.

30. Xuan D, Li G, Cai Q, et al. APOBEC3 deletion polymorphism is associated with breast cancer risk among women of European ancestry. Carcinogenesis 2013;34:2240-3.

31. Marouf C, Gohler S, Filho MI, et al. Analysis of functional germline variants in APOBEC3 and driver genes on breast cancer risk in Moroccan study population. BMC Cancer

Cite this article as: Ben $\mathrm{X}$, Tian D, Liang J, Wu M, Xie F, Zheng J, Chen J, Fei Q, Guo X, Weng X, Liu S, Xie X, Ying Y, Qiao G, Jing C. APOBEC3B deletion polymorphism and lung cancer risk in the southern Chinese population. Ann Transl Med 2021;9(8):656. doi: 10.21037/atm-21-989
2016;16:165.

32. Balkwill F, Mantovani A. Inflammation and cancer: back to Virchow? Lancet 2001;357:539-45.

33. Rezaei M, Hashemi M, Hashemi SM, et al. APOBEC3 Deletion is Associated with Breast Cancer Risk in a Sample of Southeast Iranian Population. Int J Mol Cell Med 2015;4:103-8.

34. Long J, Delahanty RJ, Li G, et al. A common deletion in the APOBEC3 genes and breast cancer risk. J Natl Cancer Inst 2013;105:573-9.

35. Gohler S, Da Silva Filho MI, Johansson R, et al. Impact of functional germline variants and a deletion polymorphism in APOBEC3A and APOBEC3B on breast cancer risk and survival in a Swedish study population. J Cancer Res Clin Oncol 2016;142:273-6.

36. Liu J, Sieuwerts AM, Look MP, et al. The $29.5 \mathrm{~kb}$ APOBEC3B Deletion Polymorphism Is Not Associated with Clinical Outcome of Breast Cancer. PLoS One 2016;11:e0161731.

37. Cohen AJ, Brauer M, Burnett R, et al. Estimates and 25year trends of the global burden of disease attributable to ambient air pollution: an analysis of data from the Global Burden of Diseases Study 2015. Lancet 2017;389:1907-18.

38. Thorgeirsson TE, Geller F, Sulem P, et al. A variant associated with nicotine dependence, lung cancer and peripheral arterial disease. Nature 2008;452:638-42.

39. Dong J, Hu Z, Wu C, et al. Association analyses identify multiple new lung cancer susceptibility loci and their interactions with smoking in the Chinese population. Nat Genet 2012;44:895-9.

40. Jung KJ, Jeon C, Jee SH. The effect of smoking on lung cancer: ethnic differences and the smoking paradox. Epidemiol Health 2016;38:e2016060.

(English Language Editor: C. Betlazar-Maseh) 


\section{Supplementary}

Table S1 Correlation between APOBEC3B and clinical pathology parameters in NSCLC

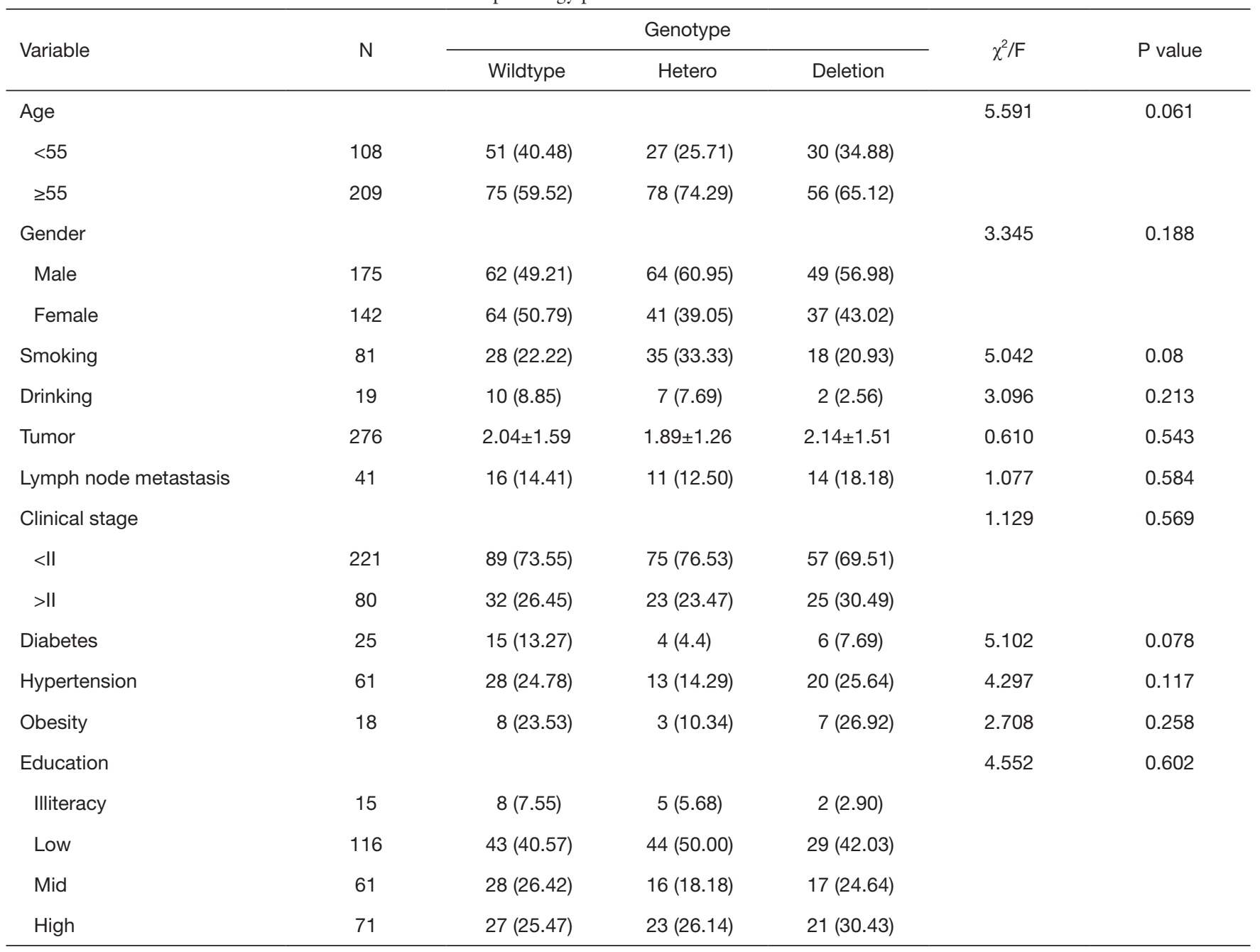

